\title{
Robust fault detection and isolation based on zonotopic unknown input observers for discrete-time descriptor systems
}

\author{
Ye Wang ${ }^{\mathrm{a}}$, Vicenç Puig ${ }^{\mathrm{b}, *}$, Feng $\mathrm{Xu}^{\mathrm{c}}$, Gabriela Cembrano ${ }^{\mathrm{b}, \mathrm{d}}$

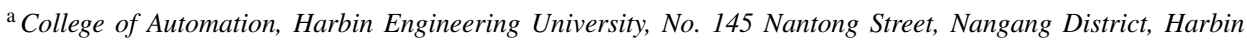 \\ 150001, PR China \\ ${ }^{\mathrm{b}}$ Advanced Control Systems (SAC) Research Group at Institut de Robòtica i Informàtica Industrial (IRI), \\ CSIC-UPC, Automatic Control Department, Universitat Politècnica de Catalunya-BarcelonaTech (UPC), C/ Llorens \\ i Artigas 4-6, Barcelona 08028, Spain \\ ${ }^{\mathrm{c} C e n t e r}$ for Artificial Intelligence and Robotics, Graduate School at Shenzhen, Tsinghua University, Shenzhen \\ 518055, PR China \\ ${ }^{\mathrm{d}}$ Cetaqua, Water Technology Centre, Ctra. d'Esplugues 75, Cornellà de Llobregat, Barcelona 08940, Spain
}

Received 5 December 2017; received in revised form 22 March 2019; accepted 10 April 2019

Available online 22 May 2019

\begin{abstract}
In this paper, we propose a robust fault detection and isolation (FDI) strategy based on zonotopic unknown input observers (UIOs) for discrete-time descriptor linear time-varying (LTV) systems subject to uncertainties and additive actuator faults. System uncertainties including state disturbances and measurement noise are unknown but bounded by predefined zonotopes. The uncertain state estimations and constructed residuals for robust FDI are propagated in a sequence of zonotopes. Based on a defined performance criterion, the fault detection (FD) observer gain is designed to be robust against uncertainties and meanwhile sensitive to faults. The explicit computational method for the FD observer gain is derived. In addition to include fault isolation, a bank of zonotopic UIOs are employed. Finally, we apply the proposed method into two case studies to show its effectiveness.
\end{abstract}

(C) 2019 The Franklin Institute. Published by Elsevier Ltd. All rights reserved.

\footnotetext{
* Corresponding author.

E-mail addresses: yewang@hrbeu.edu.cn (Y. Wang), vicenc.puig@upc.edu (V. Puig), xu.feng@sz.tsinghua.edu.cn (F. Xu), cembrano@iri.upc.edu (G. Cembrano).
} 


\section{Introduction}

Fault detection and isolation (FDI) has attracted a lot of attention during the past two decades. An FDI module as a key part of a fault-tolerant control system is also necessary for maintaining the whole system under safe operations when some elements (i.e. actuators and sensors) malfunction [1]. The aim of an FDI strategy is to detect the occurred faults and locate them in the monitored system [2,3]. Different FDI strategies have been widely studied, such as methods based on parity space, observers and parity equations [2,4-6]. Robust FDI aims at minimizing the sensitivity to uncertainties (such as modeling errors, process disturbances, measurement noise as so on) while maximizing the fault sensitivity to achieve good FDI performance. With this aim, different approaches have been studied. One category relies on the use of robust control theory tools, for instance using $H_{\infty}$ and $H_{-}$norms (see e.g. [2,710]). Among robust approaches, a standard method is based on an adaptive threshold for the decision making of the FDI alarm. The over-approximation of the decision-making threshold may lead to wrong FDI results. Alternatively, another category of FDI approaches is built under a set-theoretic framework, such as [11-13]. System uncertainties are considered as unknown but bounded in predefined sets (intervals, zonotopes and polytopes) and the resulting uncertain states and generated residuals are propagated also in bounded sets $[14,15]$. Due to the simple computational load, zonotopes are usually chosen as the geometrical sets for bounding uncertain states or residuals [16,17]. Under this framework, robustness and fault sensitivity of the FDI strategy are achieved by checking the consistency between the system model and the system measurements [18].

Unknown input observer (UIO) is a well-known approach for designing a robust FDI strategy that can be achieved by generating residuals with decoupled unknown inputs [7]. The design of UIO has been well developed for a variety of systems with different structures, such as linear dynamical systems [7,19], fuzzy systems [20-22], as well as descriptor systems [23]. In the design of UIO for implementing FDI strategy, the robustness and fault sensitivity are also taken into account. Some preliminary results show the potential of linking UIO and the set-theoretic framework as discussed in [24], where unknown inputs are divided into two groups: one can be decoupled by using UIO transformation matrices; the other cannot be decoupled but bounded using invariant sets. Besides, an extension to robust fault detection and isolation based on set-theoretic UIO has been studied in [25,26].

For many physical systems, the system model cannot be built only by differential/difference equations to describe system dynamics. Due to mass and energy balance in fluid or energy distribution networks, the model also includes algebraic equations. Such systems are called descriptor systems, also known as singular or differential/difference-algebraic systems. The descriptor system modeling approaches have been already used in a large amount of applications, such as water networks [27], chemical processes [28], aircraft [29], electrical circuits [30] and so on. For such systems, a suitable robust FDI strategy as well as fault-tolerant capabilities are required.

\subsection{Contribution}

The main contribution of this paper is to propose a robust FDI strategy for discrete-time descriptor linear time-varying (LTV) systems. The considered descriptor system is affected by uncertainties (system disturbances and measurement noise) and actuator faults. Moreover, system uncertainties are assumed to be unknown but bounded by given zonotopes. Under the 
set-theoretic framework proposed in our previous work [17], uncertain states and generated residuals are also bounded by zonotopes. In this paper, using a decomposition structure of zonotopic observer and a defined performance criterion, the observer gain for robust fault detection (FD) is designed to be robust against uncertainties while still sensitive to faults. The explicit algebraic solution for designing the FD gain is presented. In order to include fault isolation (FI), the robust FD strategy is extended using a bank of zonotopic UIOs. For each zonotopic UIO, a single actuator fault is regarded as an unknown input that can be decoupled. Therefore, the observer gain is designed to be robust against uncertainties and meanwhile sensitive to the remaining faults, which can be implemented by maximizing and minimizing the size of the corresponding zonotopes. Finally, we test different observer gains and the proposed FDI strategies in two case studies.

\subsection{Outline}

The paper structure begins with some preliminary results including definitions, properties and notations are introduced in Section 2. The problem statement is formulated in Section 3. The main results including zonotopic UIO design and robust FDI strategy are presented in Section 4. Two case studies are provided in Section 5. Finally, conclusions are drawn in Section 6.

\section{Preliminaries}

\subsection{Notation and linear algebra}

We denote $I_{r}$ as an identity matrix with $r$ dimension and the index $r$ may be dropped when the dimension is implied. For a matrix $X$, we use $X^{\top}, X_{\dagger}^{\dagger}, \operatorname{rank}(X)$ and $\operatorname{tr}(X)$ to denote the transpose, pseudo inverse, matrix rank and trace of $X$, respectively. Besides, we use $\operatorname{det}(X(z))$ to denote the determinant of $X$, and $\operatorname{deg} \operatorname{det}(X)$ to denote the degree of the determinant of $X$, both on the variable $z$. We also use $\lambda(X)$ to denote the set of eigenvalues of $X$. We denote the Minkowski sum and the linear image as $\oplus$ and $\odot$, respectively.

Given a symmetric and positive definite matrix $W \in \mathbb{R}^{n \times n}, W=W^{\top} \succ 0$ and $X \in \mathbb{R}^{n \times r}$, the weighted Frobenius norm of $X$ is defined by $\|X\|_{F, W}=\sqrt{\operatorname{tr}\left(X^{\top} W X\right)}$ and if $W=I_{n}$, it means no weight. We denote $x=\operatorname{vec}(X) \in \mathbb{R}^{n r \times 1}$ as the vectorization of $X \in \mathbb{R}^{n \times r}$ formed by stacking the columns of $X$ into a single column vector $x$, and $X=\operatorname{vec}^{-1}(x)$ as the inverse vectorization. For two matrices $X$ and $Y$, it holds

$\operatorname{tr}\left(X^{\top} Y\right)=\operatorname{vec}(X)^{\top} \operatorname{vec}(Y)=\operatorname{vec}(Y)^{\top} \operatorname{vec}(X)$.

The Kronecker product of $X$ and $Y$ is denoted by $X \otimes Y$. Consider matrices $A, B$ and $X$, the following properties hold:

$$
\begin{aligned}
\operatorname{vec}(A X B) & =\left(B^{\top} \otimes A\right) \operatorname{vec}(X), \\
\operatorname{vec}(A B) & =(I \otimes A) \operatorname{vec}(B) .
\end{aligned}
$$

\subsection{Zonotopes}

We now introduce some definitions and properties related to zonotopes and set operations as follows. 
Definition 1 (Zonotope). An $r$-order zonotope $\mathcal{Z} \subset \mathbb{R}^{n}$ in $n$-dimensional space is defined by $\mathcal{Z}=\langle p, H\rangle=\left\{p+H z, z \in \mathbf{B}^{r}\right\}$,

where $p \in \mathbb{R}^{n}$ is the center, $H \in \mathbb{R}^{n \times r}$ is the generator matrix, and $\mathbf{B}^{r}=[-1,+1]^{r} \subset \mathbb{R}^{r}$ is an $r$-order hypercube.

Definition 2 (Interval Hull). Given a zonotope $\mathcal{Z}=\langle p, H\rangle \subset \mathbb{R}^{n}$, the interval hull $r s(H) \in$ $\mathbb{R}^{n \times n}$ is defined as an aligned minimum box, where $r s(H)$ is a diagonal matrix with diagonal elements of $r s(H)_{i, i}=\sum_{j=1}^{r}\left|H_{i, j}\right|$ for $i=1,2, \ldots, n$.

Definition 3 ( $F_{W}$-radius). Given a zonotope $\mathcal{Z}=\langle p, H\rangle \subset \mathbb{R}^{n}$ and a symmetric and positive definite matrix $W \in \mathbb{R}^{n \times n}$, the $F_{W}$-radius is defined by the weighted Frobenius norm of $H$ as $\|H\|_{F, W}$, where $\|H\|_{F, W}^{2}=\operatorname{tr}\left(H^{\top} W H\right)=\operatorname{tr}\left(W H H^{\top}\right)$.

Definition 4 (Covariation). Given a zonotope $\mathcal{Z}=\langle p, H\rangle \subset \mathbb{R}^{n}$, the covariation is defined by $P=H H^{\top}$.

For zonotopes, we have the following set properties:

$$
\begin{aligned}
\left\langle p_{1}, H_{1}\right\rangle \oplus\left\langle p_{2}, H_{2}\right\rangle & =\left\langle p_{1}+p_{2},\left[\begin{array}{ll}
H_{1} & H_{2}
\end{array}\right]\right\rangle, \\
L \odot\langle p, H\rangle & =\langle L p, L H\rangle, \\
\langle p, H\rangle & \subset\langle p, r s(H)\rangle,
\end{aligned}
$$

where $L$ is a matrix of appropriate dimension.

For a zonotope $\mathcal{Z}=\langle p, H\rangle \subset \mathbb{R}^{n}$, the reduction operator is denoted as $\downarrow_{q, W}(H)$, where $q$ specifies the maximum number of column of $H$ and $W \in \mathbb{R}^{n \times n}, W=W^{\top} \succ 0$ is a weighting matrix of appropriate dimension. The inclusion property holds: $\langle p, H\rangle \subset\left\langle p, \downarrow_{q, W}(H)\right\rangle$. As introduced in [31], $\downarrow_{q, W}(H)$ can be obtained by the following procedure:

- Sort the column of segment matrix $H$ on decreasing order: $\downarrow_{W}(H)=\left[h_{1}, h_{2}, \ldots, h_{r}\right]$, $\left\|h_{j}\right\|_{W}^{2} \geq\left\|h_{j+1}\right\|_{W}^{2}$, where $\left\|h_{j}\right\|_{W}$ is the weighted 2-norm of $h_{j}$.

- Take the first $q$-column of $\downarrow_{W}(H)$ and enclose a set $H_{<}$generated by remaining columns into a smallest box (interval hull) computed by using $r s(\cdot)$ :

$$
\begin{aligned}
& \text { If } r \leq q \text {, then } \downarrow_{q, W}(H)=\downarrow_{W}(H), \\
& \text { Else } \downarrow_{q, W}(H)=\left[H_{>}, r s\left(H_{<}\right)\right] \in \mathbb{R}^{n \times q}, \\
& \quad H_{>}=\left[h_{1}, \ldots, h_{q}\right], H_{<}=\left[h_{q+1}, \ldots, h_{r}\right] .
\end{aligned}
$$

\section{Problem statement}

Consider the discrete-time descriptor LTV systems with additive actuator faults as

$E x(k+1)=A(k) x(k)+B(k) u(k)+D_{w}(k) w(k)+F(k) f(k)$,

$y(k)=C x(k)+D_{v} v(k)$,

where $x \in \mathbb{R}^{n_{x}}, u \in \mathbb{R}^{m}$ and $y \in \mathbb{R}^{n_{y}}$ denote the state, known input and output vectors, respectively. $w \in \mathbb{R}^{m_{w}}$ and $v \in \mathbb{R}^{m_{v}}$ denote the state disturbance vector and the measurement noise vector. $f \in \mathbb{R}^{m}$ denotes the normalized additive fault vector. $A(k) \in \mathbb{R}^{n_{x} \times n_{x}}, B(k) \in \mathbb{R}^{n_{x} \times m}$, $D(k) \in \mathbb{R}^{n_{x} \times m_{d}}, D_{w}(k) \in \mathbb{R}^{n_{x} \times m_{w}}$, and $F(k) \in \mathbb{R}^{n_{x} \times m}, \forall k \in \mathbb{N}$ are known time-varying system matrices. $C \in \mathbb{R}^{n_{y} \times n_{x}}$ and $D_{v} \in \mathbb{R}^{n_{y} \times m_{v}}$ are known constant system matrices. Besides, 
$E \in \mathbb{R}^{n_{x} \times n_{x}}$ satisfies $\operatorname{rank}(E) \leq n_{x}, \forall k \in \mathbb{N}$. In particular, when $\operatorname{rank}(E)=n_{x}$, Eq. (1) is equivalent to a dynamical system.

Assumption 1. The descriptor LTV system (1) is assumed to be admissible with known input vector $u(k), \forall k \in \mathbb{N}$, that is, regular, causal and stable [32].

Following the basic FDI framework [7, Chapter 6.2] and [2, Chapter 3.5], the actuator fault $f$ is modeled in an additive form with the input vector $u$. To develop a robust FDI strategy, the fault vector $f(k), \forall k \in \mathbb{N}$ can be rewritten in an element-wise form as

$f(k)=\left[f_{1}(k), \ldots, f_{i}(k), \ldots, f_{m}(k)\right]^{\top}, \quad \forall k \in \mathbb{N}$,

where the element $f_{i}(k)$ with $i=1, \ldots, m$ in the fault vector $f(k)$ corresponds to the $i$-th actuator fault at time step $k$. Then, the descriptor system (1) can be rewritten as

$E x(k+1)=A(k) x(k)+B(k) u(k)+D_{w}(k) w(k)+F_{i}(k) f_{i}(k)+\bar{F}_{i}(k) \bar{f}_{i}(k)$,

$y(k)=C x(k)+D_{v} v(k)$,

where $F_{i}(k)$ denotes the corresponding fault magnitude matrix on the $i$ th actuator, $\bar{f}_{i}(k)=$ $f(k) \backslash f_{i}(k)$ is the fault vector $f(k)$ excluding the $i$ th element and $\bar{F}_{i}(k)=F(k) \backslash F_{i}(k)$ is the matrix obtained by removing ith column from the fault magnitude matrix $F(k)$ at time step $k$.

According to $[33,34]$, the following assumption is considered in the design of the observer for the system (1).

Assumption 2. Matrices $E$ and $C$ satisfy the following rank condition:

$\operatorname{rank}\left(\left[\begin{array}{c}E \\ C\end{array}\right]\right)=n_{x}$.

Thus, from the condition (4), there always exist two matrices $T \in \mathbb{R}^{n_{x} \times n_{x}}$ and $N \in \mathbb{R}^{n_{x} \times n_{y}}$ such that

$T E+N C=I$,

and the explicit solution of $T$ and $N$ can be found in [35, (16) and (17)] as follows:

$T=\Psi \alpha_{T}, N=\Psi \alpha_{N}$,

where $\Psi=\left[\begin{array}{l}E \\ C\end{array}\right]^{\dagger}+\Sigma\left(I_{n_{x}+n_{y}}-\left[\begin{array}{l}E \\ C\end{array}\right]\left[\begin{array}{l}E \\ C\end{array}\right]^{\dagger}\right), \alpha_{T}=\left[\begin{array}{c}I_{n_{x}} \\ 0\end{array}\right], \alpha_{N}=\left[\begin{array}{c}0 \\ I_{n_{y}}\end{array}\right]$ and an arbitrary matrix $\Sigma \in \mathbb{R}^{n_{x} \times\left(n_{x}+n_{y}\right)}$.

Assumption 3. The initial state vector is assumed to be bounded in the initial zonotope $x(0) \in \mathcal{X}(0)=\langle p(0), H(0)\rangle$ and the system disturbances and measurement noise are assumed to be unknown but bounded by the centered zonotopes:

$w(k) \in \mathcal{W}=\left\langle 0, I_{m_{w}}\right\rangle, v(k) \in \mathcal{V}=\left\langle 0, I_{m_{v}}\right\rangle, \forall k \in \mathbb{N}$.

Assumption 4. The normalized fault vector $f(k)$ is assumed to be unknown but bounded by the centered zonotope $f \in \mathcal{F}=\left\langle 0, I_{m}\right\rangle, \forall k \in \mathbb{N}$ and its magnitude is given by the distribution matrix $F(k)$.

In this work, the uncertain states are estimated by a zonotope considering that all the uncertainties are also bounded by zonotopes. Based on a recursive procedure, estimation 
errors and uncertainties are also propagated using set operations. We would like to design a zonotopic UIO of the descriptor LTV system (1) and (3) to implement robust FDI. The objectives for the zonotopic UIO design are summarized as follows:

(i) Robust FD: For the descriptor LTV system (1), a zonotopic UIO with an observer gain for robust FD is designed to minimize the effects of bounded uncertainties and meanwhile to maximize the fault sensitivity on actuator faults.

(ii) Robust FI: For the descriptor LTV system (1) in the representation of Eq. (3), a bank of zonotopic UIOs for robust FI are designed. The observer gain of the $i$ th zonotopic UIO is designed to remove the effect of the corresponding actuator fault $f_{i}$, to maximize the fault sensitivity on the remaining faults $\bar{f}_{i}$, and meanwhile to minimize the effects of bounded uncertainties.

\section{Main results}

In this section, we present a set-based robust FDI strategy for discrete-time descriptor LTV systems. We first introduce the zonotopic UIO structure for discrete-time descriptor LTV systems. Then, the observer gain is designed based on the proposed objectives in the previous section.

\subsection{Zonotopic UIO structure of descriptor LTV systems}

Considering Assumption 2, we can always find a pair of matrices $T \in \mathbb{R}^{n_{x} \times n_{x}}$ and $N \in$ $\mathbb{R}^{n_{x} \times n_{y}}$ such that (5) holds. From the system (1), the descriptor dynamics can be transformed into

$$
\begin{aligned}
x(k+1)= & T A(k) x(k)+T B(k) u(k)+T D_{w}(k) w(k) \\
& +T F(k) f(k)+N y(k+1)-N D_{v} v(k+1) .
\end{aligned}
$$

According to [24,36,37], we consider a basic UIO structure as

$$
z(k+1)=M(k) z(k)+K(k) u(k)+G(k) y(k),
$$

$\hat{x}(k)=z(k)+N y(k)$,

$\hat{y}(k)=C \hat{x}(k)$,

where $z \in \mathbb{R}^{n_{x}}, \hat{x} \in \mathbb{R}^{n_{x}}$ and $\hat{y} \in \mathbb{R}^{n_{y}}$ denote vectors of the observer state, the estimated state and output. Besides, $M(k) \in \mathbb{R}^{n_{x} \times n_{x}}, K(k) \in \mathbb{R}^{n_{x} \times n_{u}}$, and $G(k) \in \mathbb{R}^{n_{x} \times n_{y}}$ are time-varying matrices to be designed. In particular, $G(k)$ is the time-varying observer gain of the UIO (9).

Let us define the state estimation error as $e(k)=x(k)-\hat{x}(k)$. From Eq. (9b), we have $e(k)=x(k)-\hat{x}(k)=x(k)-z(k)-N y(k)$. From Eq. (9) at time step $k+1$, we can derive

$$
\begin{aligned}
e(k+1) & =x(k+1)-\hat{x}(k+1) \\
& =x(k+1)-z(k+1)-N y(k+1) .
\end{aligned}
$$

By substituting $x(k+1)$ by Eq. (8) and introducing $e(k)$ in the above equation, we obtain the state estimation error dynamics as 


$$
\begin{aligned}
e(k+1)= & M(k) e(k)+(T A(k)-G(k) C-M(k)) x(k) \\
& +(T B(k)-K(k)) u(k)+M(k) N y(k) \\
& +T D_{w}(k) w(k)+T F(k) f(k)-G(k) D_{v} v(k)-N D_{v} v(k+1) .
\end{aligned}
$$

For the case of $f \equiv 0$, we now define recursively the zonotopic UIO of the descriptor LTV system (1). Consider that the state vector $x(k)$ of the descriptor LTV system (1) satisfies the inclusion $x(k) \in \mathcal{X}(k)=\langle p(k), H(k)\rangle$ at time step $k \in \mathbb{N}$, which also satisfies the initial state vector $x(0) \in\langle p(0), H(0)\rangle$ at time step $k=0$.

Theorem 1 (Zonotopic UIO structure of descriptor LTV systems). Consider the admissible descriptor LTV system (1) with $f \equiv 0$ and $x(k) \in \mathcal{X}(k)=\langle p(k), H(k)\rangle$ at time step $k \in \mathbb{N}$. The zonotopic UIO of the descriptor system (1) can be recursively defined by $x(k+1) \in$ $\mathcal{X}(k+1)=\langle p(k+1), H(k+1)\rangle$, where

$$
\left\{\begin{array}{l}
p(k+1)=(T A(k)-G(k) C) p(k)+T B(k) u(k)+G(k) y(k)+N y(k+1), \\
H(k+1)=\left[R(k), T D_{w}(k),-G(k) D_{v},-N D_{v}\right],
\end{array}\right.
$$

with $R(k)=(T A(k)-G(k) C) \bar{H}(k)$ and $\bar{H}(k)=\downarrow_{q, W}(H(k))$.

Proof. Consider $x(k) \in\langle p(k), H(k)\rangle$ at time step $k \in \mathbb{N}$. According to the inclusion property in Section 2.2, $\langle p(k), H(k)\rangle \subset\langle p(k), \bar{H}(k)\rangle$ holds. By setting $\hat{x}(k)=p(k)$, we have $e(k)=$ $x(k)-\hat{x}(k) \in\langle 0, H(k)\rangle \subset\langle 0, \bar{H}(k)\rangle$. Therefore, at time step $k+1$, we have $x(k+1)=$ $e(k+1)+\hat{x}(k+1)$.

From $e(k+1)$ in Eq. (10), let us choose

$M(k)=T A(k)-G(k) C$,

$K(k)=T B(k)$.

Taking into account $f \equiv 0$, Eq. (10) becomes

$$
\begin{aligned}
e(k+1)= & (T A(k)-G(k) C) e(k)+(T A(k)-G(k) C) N y(k) \\
& +T D_{w}(k) w(k)-G(k) D_{v} v(k)-N D_{v} v(k+1) .
\end{aligned}
$$

From Eq. (9), we can derive

$$
\begin{aligned}
\hat{x}(k+1)= & (T A(k)-G(k) C) p(k)+T B(k) u(k) \\
& +(G(k)-(T A(k)-G(k) C) N) y(k)+N y(k+1) .
\end{aligned}
$$

Considering $e(k) \in\langle 0, \bar{H}(k)\rangle, \quad w(k) \in \mathcal{W}=\left\langle 0, I_{m_{w}}\right\rangle$ and $v(k), v(k+1) \in \mathcal{V}=\left\langle 0, I_{m_{v}}\right\rangle$, $\forall k \in \mathbb{N}$, from $x(k+1)=e(k+1)+\hat{x}(k+1)$, we derive

$$
\begin{aligned}
x(k+1) \in & \langle p(k+1), H(k+1)\rangle \\
= & ((T A(k)-G(k) C) \odot\langle 0, \bar{H}(k)\rangle) \\
& \oplus\langle(T A(k)-G(k) C) N y(k), 0\rangle \\
& \oplus\left(T D_{w}(k) \odot\left\langle 0, I_{m_{w}}\right\rangle\right) \oplus\left(-G(k) D_{v} \odot\left\langle 0, I_{m_{v}}\right\rangle\right) \\
& \oplus\left(-N D_{v} \odot\left\langle 0, I_{m_{v}}\right\rangle\right) \oplus\langle\hat{x}(k+1), 0\rangle .
\end{aligned}
$$

Thus, using the zonotope properties in Section 2.2, we obtain $p(k+1)$ and $H(k+1)$ as in Eq. (11). 
Remark 1. Note that the zonotope $\mathcal{X}(k)=\langle p(k), H(k)\rangle$ is used for bounding $x(k), \forall k \in \mathbb{N}$ with $f \equiv 0$ while the estimated state $\hat{x}(k)$ in Eq. (9) only determines the nominal value and the estimation error is omitted in the formulation of Eq. (9). According to the proof of Theorem 1, from $x(k) \in\langle p(k), H(k)\rangle$, we know $p(k)=\hat{x}(k)$ and the state estimation error $e(k)=x(k)-\hat{x}(k) \in\langle 0, H(k)\rangle$.

Remark 2. Considering Assumption 3 and $f \equiv 0$, from the output Eq. (1b), for $x(k) \in \mathcal{X}(k)=$ $\langle p(k), H(k)\rangle, \forall k \in \mathbb{N}$, we can derive the output zonotope $\mathcal{Y}(k)=\left\langle p_{y}(k), H_{y}(k)\right\rangle$, where

$$
\begin{aligned}
y(k) & \in\left\langle p_{y}(k), H_{y}(k)\right\rangle \\
& =(C \odot\langle p(k), H(k)\rangle) \oplus\left(D_{v} \odot\left\langle 0, I_{m_{v}}\right\rangle\right) \\
& =\left\langle C p(k),\left[C H(k), D_{v}\right]\right\rangle .
\end{aligned}
$$

Since $p(k)=\hat{x}(k)$, from the output zonotope $\mathcal{Y}(k)=\left\langle p_{y}(k), H_{y}(k)\right\rangle$, we also know $\hat{y}(k)=$ $p_{y}(k)=C p(k)$ and the output estimation error $\epsilon(k)=y(k)-\hat{y}(k) \in\left\langle 0, H_{y}(k)\right\rangle$.

To implement an FDI strategy, let us define the residual zonotope $\mathcal{R}(k)=y(k) \oplus(-\mathcal{Y}(k))$. We present the explicit computational result of this residual zonotope in the following.

Corollary 1. Consider the admissible descriptor LTV system (1) and $x(k) \in \mathcal{X}(k)=$ $\langle p(k), H(k)\rangle, \forall k \in \mathbb{N}$. The residual zonotope is given by $\mathcal{R}(k)=\left\langle p_{r}(k), H_{r}(k)\right\rangle$, where

$$
\left\{\begin{array}{l}
p_{r}(k)=y(k)-C p(k), \\
H_{r}(k)=\left[-C H(k), \quad-D_{v}\right] .
\end{array}\right.
$$

Proof. Based on Theorem $1, x(k) \in\langle p(k), H(k)\rangle$ can be computed recursively, $\forall k \in \mathbb{N}$. According to the definition of $\mathcal{R}(k)$, it follows

$$
\begin{aligned}
\mathcal{R}(k) & =\left\langle p_{r}(k), H_{r}(k)\right\rangle \\
& =y(k) \oplus\left\langle-C p(k),\left[-C H(k),-D_{v}\right]\right\rangle .
\end{aligned}
$$

Thus, using the zonotope properties in Section 2.2, we obtain (13).

The output Eq. (1b) can be rewritten as $0=y(k)-C x(k)-D_{v} v(k)$. Taking into account that $v(k) \in\left\langle 0, I_{m_{v}}\right\rangle$ and $x(k) \in \mathcal{X}(k)=\langle p(k), H(k)\rangle$, if no fault has occurred at time step $k$, then the following condition holds:

$0 \in \mathcal{R}(k)$.

To analyze the effects of occurred actuator faults in the defined state or residual zonotopes above, we consider the normalized fault vector $f(k) \in \mathcal{F}, \forall k \in \mathbb{N}$, i.e., the magnitude of the fault vector $f(k)$ is stored in the matrix $F(k)$. Therefore, we present the decomposed zonotopic UIO structure for the descriptor system in the presence of faults considering $f(k) \in \mathcal{F}, \forall k \in \mathbb{N}$ in the following theorem.

Theorem 2 (Zonotopic UIO decomposition of descriptor LTV systems). Consider the admissible descriptor LTV system (1) with $f(k) \in \mathcal{F}$ and $x(k) \in\left\{\left\langle p_{e}(k), H_{e}(k)\right\rangle \oplus\left\langle p_{f}(k), H_{f}(k)\right\rangle\right\}$, $\forall k \in \mathbb{N}$. The zonotopic UIO affected by actuator faults can be recursively defined in the decomposition form as $x(k+1) \in\left\{\left\langle p_{e}(k+1), H_{e}(k+1)\right\rangle \oplus\left\langle p_{f}(k+1), H_{f}(k+1)\right\rangle\right\}$, where

$$
\left\{\begin{array}{l}
p_{e}(k+1)=(T A(k)-G(k) C) p_{e}(k)+T B(k) u(k)+G(k) y(k)+N y(k+1), \\
H_{e}(k+1)=\left[\begin{array}{ll}
R_{e}(k), & T D_{w}(k), \quad-G(k) D_{v}, \quad-N D_{v}
\end{array}\right],
\end{array}\right.
$$

and 
$\left\{\begin{array}{l}p_{f}(k+1)=(T A(k)-G(k) C) p_{f}(k), \\ H_{f}(k+1)=\left[(T A(k)-G(k) C) \bar{H}_{f}(k), \quad T F(k)\right],\end{array}\right.$

with $R_{e}(k)=(T A(k)-G(k) C) \bar{H}_{e}(k), \bar{H}_{e}(k)=\downarrow_{q, W}\left(H_{e}(k)\right), \bar{H}_{f}(k)=\downarrow_{q, W}\left(H_{f}(k)\right), H_{e}(k+$ 1) $\in \mathbb{R}^{n_{x} \times n_{e}}$, and $H_{f}(k+1) \in \mathbb{R}^{n_{x} \times n_{f}}$.

Proof. Consider $\quad x(k) \in\left\{\left\langle p_{e}(k), H_{e}(k)\right\rangle \oplus\left\langle p_{f}(k), H_{f}(k)\right\rangle\right\} \subset\left\{\left\langle p_{e}(k), \bar{H}_{e}(k)\right\rangle \oplus\right.$ $\left.\left\langle p_{f}(k), \bar{H}_{f}(k)\right\rangle\right\}=\left\langle\left(p_{e}(k)+p_{f}(k)\right),\left[\bar{H}_{e}(k), \bar{H}_{f}(k)\right]\right\rangle$. By setting $\hat{x}(k)=p_{e}(k)+p_{f}(k)$, we have $e(k)=x(k)-\hat{x}(k) \in\left\langle 0,\left[\bar{H}_{e}(k), \bar{H}_{f}(k)\right]\right\rangle$.

Let us choose the matrices $M(k)$ and $K(k)$ as in Eq. (12). With $w(k) \in \mathcal{W}=\left\langle 0, I_{m_{w}}\right\rangle, v(k)$, $v(k+1) \in \mathcal{V}=\left\langle 0, I_{m_{v}}\right\rangle$ and $f(k) \in \mathcal{F}=\left\langle 0, I_{m}\right\rangle$, we derive $x(k+1)=e(k+1)+\hat{x}(k+1)$ to obtain

$$
\begin{aligned}
x(k+1) \in & \left\{\left\langle p_{e}(k+1), H_{e}(k+1)\right\rangle \oplus\left\langle p_{f}(k+1), H_{f}(k+1)\right\rangle\right\} \\
= & \left((T A(k)-G(k) C) \odot\left\langle p_{e}(k)+p_{f}(k),\left[\bar{H}_{e}(k), \quad \bar{H}_{f}(k)\right]\right\rangle\right) \\
& \oplus\langle T B(k) u(k), 0\rangle \oplus\langle G(k) y(k), 0\rangle \oplus\langle N y(k+1), 0\rangle \\
& \oplus\left(T D_{w}(k) \odot\left\langle 0, I_{m_{w}}\right\rangle\right) \oplus\left(-G(k) D_{v} \odot\left\langle 0, I_{m_{v}}\right\rangle\right) \\
& \oplus\left(-N D_{v} \odot\left\langle 0, I_{m_{v}}\right\rangle\right) \oplus\left(T F(k) \odot\left\langle 0, I_{m}\right\rangle\right) .
\end{aligned}
$$

Therefore, the zonotope $\left\langle p_{e}(k+1), H_{e}(k+1)\right\rangle$ is only affected by uncertainties while the zonotope $\left\langle p_{f}(k+1), H_{f}(k+1)\right\rangle$ is only affected by faults if they are chosen as in Eqs. (15) and (16).

Corollary 2. Consider the admissible descriptor LTV system (1) with $f(k) \in \mathcal{F}$ and $x(k) \in\left\{\left\langle p_{e}(k), H_{e}(k)\right\rangle \oplus\left\langle p_{f}(k), H_{f}(k)\right\rangle\right\}, \forall k \in \mathbb{N}$. The residual zonotope $\mathcal{R}(k)=\left\langle p_{r}(k), H_{r}(k)\right\rangle$ can be decomposed into $\mathcal{R}(k)=\left\{\left\langle p_{r e}(k), H_{r e}(k)\right\rangle \oplus\left\langle p_{r f}(k), H_{r f}(k)\right\rangle\right\}$, where

$\left\{\begin{array}{l}p_{r e}(k)=y(k)-C p_{e}(k), \\ H_{r e}(k)=\left[-C H_{e}(k),-D_{v}\right] .\end{array}\right.$

and

$\left\{\begin{array}{l}p_{r f}(k)=-C p_{f}(k), \\ H_{r f}(k)=-C H_{f}(k) .\end{array}\right.$

Proof. The proof is straightforward based on the zonotope properties and therefore is omitted here.

From Theorem 2 and Corollary 2, we have divided the effects of system uncertainties and faults on the residual zonotopes. Specifically, the effects of uncertainties (disturbances and noise) are propagated to the zonotope $\left\langle p_{e}(k+1), H_{e}(k+1)\right\rangle$ while the effects of faults are constrained in the zonotope $\left\langle p_{f}(k+1), H_{f}(k+1)\right\rangle$. Hence, for the FD observer gain $G(k)$ design, we use the decomposed zonotopic UIO structure defined in Theorem 2 to consider robustness to uncertainties and sensitivity to actuator faults.

\subsection{Observer gain designs}

As discussed in Theorem 2, we characterize the effects of uncertainties and faults in the zonotopes $\left\langle p_{e}(k+1), H_{e}(k+1)\right\rangle$ and $\left\langle p_{f}(k+1), H_{f}(k+1)\right\rangle$ separately. Hence, the problem of designing an FD observer gain to be robust against uncertainties and to be sensitive to faults is transformed to minimizing or maximizing the size of these zonotopes. Inspired by Combastel [38], the size of a zonotope can be measured by the $F_{W}$-radius. 


\subsubsection{Optimal Kalman gain for state estimation}

For state estimation, the objective of the observer gain design is only to minimize the effects of uncertainties. For the admissible descriptor system (1) in the fault-free case $(f=0)$, the optimal Kalman observer gain can be computed in the following theorem.

Theorem 3 (Optimal Kalman gain for descriptor LTV systems). Given the zonotopic UIO structure in Eq. (11) of the admissible descriptor LTV system (1) with $f \equiv 0$ and a matrix $W \in$ $\mathbb{R}^{n_{x} \times n_{x}}, W=W^{\top} \succ 0$, the optimal time-varying Kalman gain $\bar{G}(k)=\arg \min _{G(k)} J_{s}$, where $J_{s}=\|H(k+1)\|_{F, W}^{2}$ is computed by the following procedure:

$\bar{G}(k)=T A(k) \bar{K}(k)$,

$\bar{K}(k)=L(k) S(k)^{-1}$,

$L(k)=\bar{P}(k) C^{\top}$,

$S(k)=C(k) \bar{P}(k) C^{\top}+D_{v} D_{v}^{\top}$,

with $\bar{P}(k)=\bar{H}(k) \bar{H}(k)^{\top}$.

Proof. For $x(k+1) \in\langle p(k+1), H(k+1)\rangle$ in Eq. (11), the criterion $J_{s}=\|H(k+1)\|_{F, W}^{2}=$ $\operatorname{tr}(W P(k+1))$ with $P(k+1)=H(k+1)(H(k+1))^{\top}$ is convex with respect to $G(k)$. The optimal Kalman gain $\bar{G}(k)$ satisfies $\frac{d}{d G(k)} \operatorname{tr}(W P(k+1))=0$. Hence, we compute the derivative of $J_{s}$ with respect to $G(k)$. Selecting $L(k)$ and $S(k)$ as in Eq. (19), we have

$\frac{d}{d G(k)} \operatorname{tr}\left(W G(k) S(k) G(k)^{\top}\right)-2 \frac{d}{d G(k)} \operatorname{tr}\left(W T A(k) L(k) G(k)^{\top}\right)=0$.

From the above equation, we obtain the optimal Kalman gain $\bar{G}(k)$ as in Eq. (19).

Remark 3. As discussed in [38, Theorem 5], Theorem 3 also shows that the optimal Kalman gain $\bar{G}(k)$ is independent of the weighting matrix $W$. Therefore, the matrix $W$ can be set to be freely time-varying along the time step $k \in \mathbb{N}$.

\subsubsection{FD observer gain}

To design an FD observer gain, in addition to guarantee robustness to uncertainties, we would like to maximize the fault sensitivity with respect to actuator faults, which can be realized by maximizing the $F_{W}$-radius of the zonotope $\left\langle p_{f}(k+1), H_{f}(k+1)\right\rangle$. Assume that there exist matrices $T, N$ and $G(k)$ such that the zonotopic UIO in Eq. (11) is stable. The objectives of the FD observer gain $G(k)$ can be implemented by solving the following optimization problem:

$\min _{G}\left\|H_{e}(k+1)\right\|_{F, W_{1}}^{2}$ and simultaneously $\max _{G}\left\|H_{f}(k+1)\right\|_{F, W_{2}}^{2}$,

with matrices $W_{1}, W_{2} \in \mathbb{R}^{n_{x} \times n_{x}}$ and $W_{1}=W_{1}^{\top} \succ 0, W_{2}=W_{2}^{\top} \succ 0$.

To implement the optimization problem above, we define a performance criterion as

$J_{e / f}=\frac{\left\|H_{f}(k+1)\right\|_{F, W_{1}}^{2}}{\left\|H_{e}(k+1)\right\|_{F, W_{2}}^{2}}$.

Therefore, the optimization problem (20) is converted to maximize $J_{\text {elf. }}$. In order to find the solution of the FD observer gain, we first reformulate $\left\|H_{f}(k+1)\right\|_{F, W_{1}}^{2}$ and $\left\|H_{e}(k+1)\right\|_{F, W_{2}}^{2}$ 
using the properties in Section 2.1 as follows. From Definition 3, for $\left\|H_{f}(k+1)\right\|_{F, W_{1}}^{2}$, we have

$$
\begin{aligned}
\left\|H_{f}(k+1)\right\|_{F, W_{1}}^{2} & =\operatorname{tr}\left(\left(H_{f}(k+1)\right)^{\top} W_{1} H_{f}(k+1)\right)=\operatorname{tr}\left(W_{1} H_{f}(k+1)\left(H_{f}(k+1)\right)^{\top}\right) \\
& =\operatorname{vec}\left(H_{f}(k+1)\right)^{\top} \operatorname{vec}\left(W_{1} H_{f}(k+1)\right) \\
& =\operatorname{vec}\left(H_{f}(k+1)\right)^{\top}\left(I_{n_{f}} \otimes W_{1}\right) \operatorname{vec}\left(H_{f}(k+1)\right),
\end{aligned}
$$

and from Eq. (16), we have

$\operatorname{vec}\left(H_{f}(k+1)\right)=\left[\begin{array}{c}-\left(C \bar{H}_{f}(k)^{\top} \otimes I_{n_{x}}\right. \\ 0\end{array}\right] \operatorname{vec}(G(k))+\left[\begin{array}{c}\operatorname{vec}\left(T A(k) \bar{H}_{f}(k)\right) \\ \operatorname{vec}(T F(k))\end{array}\right]$.

Selecting $\theta(k)=\operatorname{vec}(G(k))$ and

$S_{f}(k)=\left[\begin{array}{c}-\left(C \bar{H}_{f}(k)\right)^{\top} \otimes I_{n_{x}} \\ 0\end{array}\right], b_{f}(k)=\left[\begin{array}{c}\operatorname{vec}\left(T A(k) \bar{H}_{f}(k)\right) \\ \operatorname{vec}(T F(k))\end{array}\right]$,

we have

$$
\begin{aligned}
\left\|H_{f}(k+1)\right\|_{F, W_{1}}^{2} & =\left(S_{f}(k) \theta(k)+b_{f}(k)\right)^{\top}\left(I_{n_{f}} \otimes W_{1}\right)\left(S_{f}(k) \theta(k)+b_{f}(k)\right) \\
& =\left[\begin{array}{c}
\theta(k) \\
1
\end{array}\right]^{\top}\left[\begin{array}{ll}
S_{f}(k), & b_{f}(k)
\end{array}\right]^{\top}\left(I_{n_{f}} \otimes W_{1}\right)\left[S_{f}(k), \quad b_{f}(k)\right]\left[\begin{array}{c}
\theta(k) \\
1
\end{array}\right] \\
& =\bar{\theta}(k)^{\top} Q_{f}(k) \bar{\theta}(k),
\end{aligned}
$$

where $\bar{\theta}(k)=\left[\begin{array}{c}\theta(k) \\ 1\end{array}\right]$ and

$Q_{f}(k)=\left[S_{f}(k), \quad b_{f}(k)\right]^{\top}\left(I_{n_{f}} \otimes W_{1}\right)\left[S_{f}(k), \quad b_{f}(k)\right]$.

Similarly, $\left\|H_{e}(k+1)\right\|_{F}^{2}$ can be reformulated as

$\left\|H_{e}(k+1)\right\|_{F, W_{2}}^{2}=\bar{\theta}(k)^{\top} Q_{e}(k) \bar{\theta}(k)$,

where

$Q_{e}(k)=\left[S_{e}(k), \quad b_{e}(k)\right]^{\top}\left(I_{n_{e}} \otimes W_{2}\right)\left[S_{e}(k), \quad b_{e}(k)\right]$,

and

$S_{e}(k)=\left[\begin{array}{c}-\left(C \bar{H}_{e}(k)\right)^{\top} \otimes I_{n_{x}} \\ -D_{v}^{\top} \otimes I_{n_{x}} \\ 0 \\ 0\end{array}\right], b_{e}(k)=\left[\begin{array}{c}\operatorname{vec}\left(T A(k) \bar{H}_{e}(k)\right) \\ 0 \\ \operatorname{vec}\left(T D_{w}(k)\right) \\ -\operatorname{vec}\left(N D_{v}\right)\end{array}\right]$.

Then, the performance criterion $J_{\text {elf }}$ defined in Eq. (21) can be rewritten as

$J_{e / f}=\frac{\bar{\theta}(k)^{\top} Q_{f}(k) \bar{\theta}(k)}{\bar{\theta}(k)^{\top} Q_{e}(k) \bar{\theta}(k)}$.

Due to the change of variables $\bar{\theta}(k)=\left[\begin{array}{c}\theta(k) \\ 1\end{array}\right]$ and $\theta(k)=\operatorname{vec}(G(k))$, finding an FD observer gain $G$ by maximizing $J_{\text {elf }}$ in Eq. (21) is equivalent to finding $\bar{\theta}^{*}(k)$ such that $J_{e l f}$ in Eq. (24) is maximum. Then, we provide the explicit solutions of the optimal $\bar{\theta}^{*}(k)$ corresponding to the maximum $J_{e / f}^{*}$ in the following theorem. 
Theorem 4. Given the criterion $J_{\text {elf }}$ defined in Eq. (24) with respect to $\bar{\theta}(k)$ and matrices $W_{1}, W_{2} \in \mathbb{R}^{n_{x} \times n_{x}}$ with $W_{1}=W_{1}^{\top} \succ 0$ and $W_{2}=W_{2}^{\top} \succ 0$, the maximum $J_{\text {elf }}$ is the maximum generalized eigenvalue of $\left(Q_{f}(k), Q_{e}(k)\right)$ with $Q_{f}(k)$ as in Eq. (22) and $Q_{e}(k)$ as in Eq. (23), that is denoted by $J_{e / f}^{*}=\lambda_{\max }\left(Q_{f}(k), Q_{e}(k)\right)$, and the optimal $\bar{\theta}^{*}(k)$ belongs to the null space of $\left(Q_{f}(k)-J_{e / f}^{*} Q_{e}(k)\right)$, that is also the generalized eigenvector of $\left(Q_{f}(k), Q_{e}(k)\right)$ corresponding to its maximum generalized eigenvalue.

Proof. To find the optimal $\bar{\theta}^{*}(k)$ corresponding to the maximum $J_{e / f}^{*}$, we take the derivative of $J_{\text {elf }}$ in Eq. (24) with respect to $\bar{\theta}(k)$ as

$\frac{d}{d \bar{\theta}(k)} J_{e / f}=\frac{2 Q_{f}(k) \bar{\theta}(k)\left(\bar{\theta}(k)^{\top} Q_{e}(k) \bar{\theta}(k)\right)-2 Q_{e}(k) \bar{\theta}(k)\left(\bar{\theta}(k)^{\top} Q_{f}(k) \bar{\theta}(k)\right)}{\left(\bar{\theta}(k)^{\top} Q_{e}(k) \bar{\theta}(k)\right)^{2}}$,

By setting $\frac{d}{d \bar{\theta}(k)} J_{e / f}=0$, we obtain

$2 Q_{f}(k) \bar{\theta}^{*}(k)\left(\bar{\theta}^{*}(k)^{\top} Q_{e} \bar{\theta}^{*}(k)\right)-2 Q_{e}(k) \bar{\theta}^{*}(k)\left(\bar{\theta}^{*}(k)^{\top} Q_{f}(k) \bar{\theta}^{*}(k)\right)=0$,

which can be simplified into

$Q_{f}(k) \bar{\theta}^{*}(k)=\frac{\bar{\theta}^{*}(k)^{\top} Q_{f}(k) \bar{\theta}^{*}(k)}{\bar{\theta}^{*}(k)^{\top} Q_{e}(k) \bar{\theta}^{*}(k)} Q_{e}(k) \bar{\theta}^{*}(k)$.

From Eq. (24) and $\bar{\theta}^{*}(k)$ corresponding to the maximum $J_{e / f}^{*}$, we have

$Q_{f}(k) \bar{\theta}^{*}(k)=J_{e / f}^{*} Q_{e}(k) \bar{\theta}^{*}(k)$.

Therefore, Eq. (25) leads to a generalized eigenvalue problem with $J_{e / f}^{*}=$ $\lambda_{\max }\left(Q_{f}(k), Q_{e}(k)\right)$ being the maximum generalized eigenvalue and $\bar{\theta}^{*}(k)$ being the corresponding eigenvector. Besides, from Eq. (25), we can also derive $\left(Q_{f}(k)-J_{e / f}^{*} Q_{e}(k)\right) \bar{\theta}^{*}(k)=$ 0 . Hence, $\bar{\theta}^{*}(k)$ also belongs to the null space of $\left(Q_{f}(k)-J_{e / f}^{*} Q_{e}(k)\right)$.

Based on the optimal solution $\bar{\theta}^{*}(k)$, we derive the optimal FD observer gain in the following theorem.

Theorem 5 (Optimal FD observer gain for descriptor LTV systems). Given the optimal solution $\bar{\theta}^{*}(k)$ from Theorem 4 as $\bar{\theta}^{*}(k)=\left[\begin{array}{c}\tilde{\theta}^{*}(k) \\ \check{\theta}^{*}(k)\end{array}\right]$, $\tilde{\theta}^{*}(k) \in \mathbb{R}^{\left(n_{x} \cdot n_{y}\right) \times 1}$ and $\check{\theta}^{*}(k) \in \mathbb{R}$, the optimal $F D$ observer gain $G^{*}(k)$ can be computed by

$G^{*}(k)=\operatorname{vec}^{-1}\left(\frac{\tilde{\theta}^{*}(k)}{\check{\theta}^{*}(k)}\right)$.

Proof. By dividing $\check{\theta}^{*}(k)$ on both sides of Eq. (25), we have

$Q_{f}(k)\left[\begin{array}{c}\frac{\tilde{\theta}^{*}(k)}{\tilde{\theta}^{*}(k)} \\ 1\end{array}\right]=J_{e / f}^{*} Q_{e}(k)\left[\begin{array}{c}\frac{\tilde{\theta}^{*}(k)}{\tilde{\theta}^{*}(k)} \\ 1\end{array}\right]$.

Based on the structure of $\bar{\theta}(k)=\left[\begin{array}{c}\theta(k) \\ 1\end{array}\right]$, we thus obtain $G^{*}(k)$ as in Eq. (26).

Remark 4. Note that the zonotopic UIO for the descriptor LTV system (1) is defined by a dynamical form in Eq. (11). Considering the result in [38, Section 7], the stability of this zonotopic UIO with the optimal time-varying gain $G^{*}(k)$, obtained by implementing Theorem 5, can be guaranteed. 


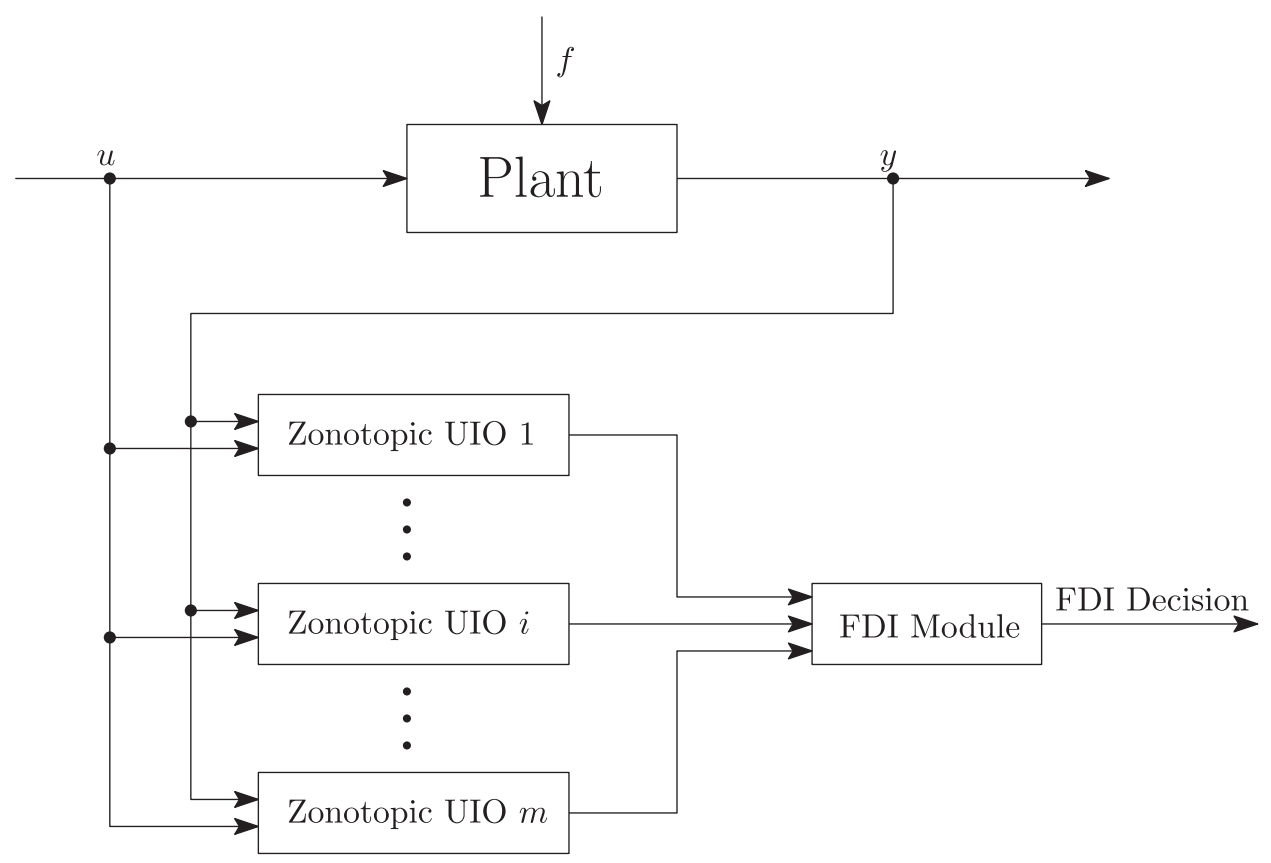

Fig. 1. The robust FDI scheme.

\subsection{Robust FDI strategy}

To include robust FI, the idea is to design a bank of zonotopic UIOs for identifying the effect from each actuator fault. From Eq. (2), the single fault is considered as an unknown input to be decoupled for the corresponding zonotopic UIO. The general robust FDI scheme is presented in Fig. 1. For the descriptor LTV system (1) with $m$ actuators, we would like to design $m$ zonotopic UIOs. By checking the residual zonotopes obtained by $m$ zonotopic UIOs, the FDI alarm can be determined by the FDI module.

From the descriptor LTV representation in Eq. (3), we treat $f_{i}(k), i=1, \ldots, m$ as an unknown input of the descriptor LTV system (3). With $f_{i}(k)$ and $\bar{f}_{i}(k), i=1, \ldots, m$, the descriptor dynamics can be reformulated as

$$
\begin{aligned}
x(k+1)= & T A(k) x(k)+T B(k) u(k)+N y(k+1) \\
& +T D_{w}(k) w(k)-N D_{v} v(k+1)+T F_{i}(k) f_{i}(k)+T \bar{F}_{i}(k) \bar{f}_{i}(k),
\end{aligned}
$$

and from Eq. (27), the state estimation error dynamics can also be reformulated as

$$
\begin{aligned}
e(k+1)= & (T A(k)-G(k) C) e(k)+(T A(k)-G(k) C) N y(k) \\
& +T D_{w}(k) w(k)-G(k) D_{v} v(k)-N D_{v} v(k+1)+T F_{i}(k) f_{i}(k)+T \bar{F}_{i}(k) \bar{f}_{i}(k) .
\end{aligned}
$$

To remove the effect of $f_{i}(k)$ and preserve the effect of $\bar{f}_{i}(k)$ in Eqs. (16) and (28), a pair of matrices $T_{i} \in \mathbb{R}^{n_{x} \times n_{x}}$ and $N_{i} \in \mathbb{R}^{n_{x} \times n_{y}}$ for the $i$ th zonotopic UIO also satisfies (5) and $T_{i} F_{i}(k)=0$,

$T_{i} \bar{F}_{i}(k) \neq 0$. 
Based on the results in [17, Eq. (5)], we present the condition for the existence of matrices $T_{i}$ and $N_{i}$ satisfying Eqs. (5) and (29a) in the following.

Assumption 5. For the descriptor LTV system (1), the matrices $E, C$ and $F_{i}(k)$ satisfy the following rank condition:

$\operatorname{rank}\left(\left[\begin{array}{c}I_{n_{x}} \otimes\left[\begin{array}{cc}E & F_{i}(k) \\ C & 0\end{array}\right] \\ \operatorname{vec}\left(\left[\begin{array}{c}I_{n_{x}} \\ 0\end{array}\right]\right)^{\top}\end{array}\right]\right)=n_{x} \cdot\left[\begin{array}{cc}E & F_{i}(k) \\ C & 0\end{array}\right], \forall k \in \mathbb{N}$.

Therefore, from the proof of Theorem 1, we know $x(k+1)=e(k+1)+\hat{x}(k+1)$. In Eq. (28), the effect of $f_{i}$ is removed in $e(k+1)$ by using the matrix $T_{i}$ with $T_{i} F_{i}(k)=0$ and meanwhile the effect of $\bar{f}_{i}(k)$ is preserved. Besides, for designing the $i$ th observer gain, considering $\bar{f}_{i}(k) \in \mathcal{F}_{i}=\left\langle 0, I_{m-1}\right\rangle$, we replace $T$ by $T_{i}$ and $F(k)$ by $\bar{F}_{i}(k)$ in Eq. (16). Following the procedure in the previous section, the optimal observer gain $G_{i}^{*}(k)$ for robust FDI can be obtained.

After having $m$ zonotopic UIOs, at each time step, a sequence of residual zonotopes $\left\langle p_{r, i}(k)\right.$, $\left.H_{r, i}(k)\right\rangle, i=1, \ldots, m$ can be generated based on Corollary 1 . Then, a fault can be determined in the FDI module. The logics of the FDI module are proposed as follows.

The logics of the FDI module:

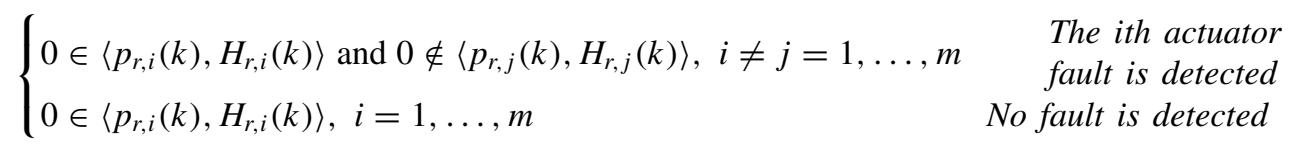

Note that we can also decouple $m-1$ actuator faults as unknown inputs by finding suitable $T_{i}$ and $N_{i}$. For the remaining fault that is not decoupled, if $0 \notin\left\langle p_{r, i}, H_{r, i}\right\rangle, i=1, \ldots, m$, then it can be detected.

We now summarize the robust FDI strategy in Algorithm 1 considering a simulation horizon of $\Gamma$.

Remark 5. The proposed robust FDI strategy based on zonotopic UIOs can also be applied to standard dynamical systems, that is when $\operatorname{rank}(E)=n_{x}, \forall k \in \mathbb{N}$.

\section{Case studies}

\subsection{A numerical example}

To compare the FD observer gain obtained with the proposed approach with zonotopic Kalman observer gain, we consider the descriptor LTV system (1) with

$$
\begin{aligned}
& E=\left[\begin{array}{llll}
1 & 0 & 0 & 0 \\
0 & 1 & 0 & 0 \\
0 & 0 & 1 & 0 \\
0 & 0 & 0 & 0
\end{array}\right], A(k)=\left[\begin{array}{cccc}
0.5 & 0.3 \sin (0.4 k) & 0 & 0 \\
0 & 0.3 & 0 & 0 \\
0 & 0 & 0.6 & 0 \\
0 & -0.5 & -0.5 & 0.8
\end{array}\right], B=\left[\begin{array}{c}
0.1 \\
1 \\
-0.1 \\
-1
\end{array}\right], \\
& C=\left[\begin{array}{llll}
0 & 1 & 0 & 0 \\
0 & 0 & 1 & 0 \\
0 & 0 & 0 & 1
\end{array}\right], D_{w}=0.005 I_{4}, D_{v}=0.01 I_{3}, F=2 B,
\end{aligned}
$$




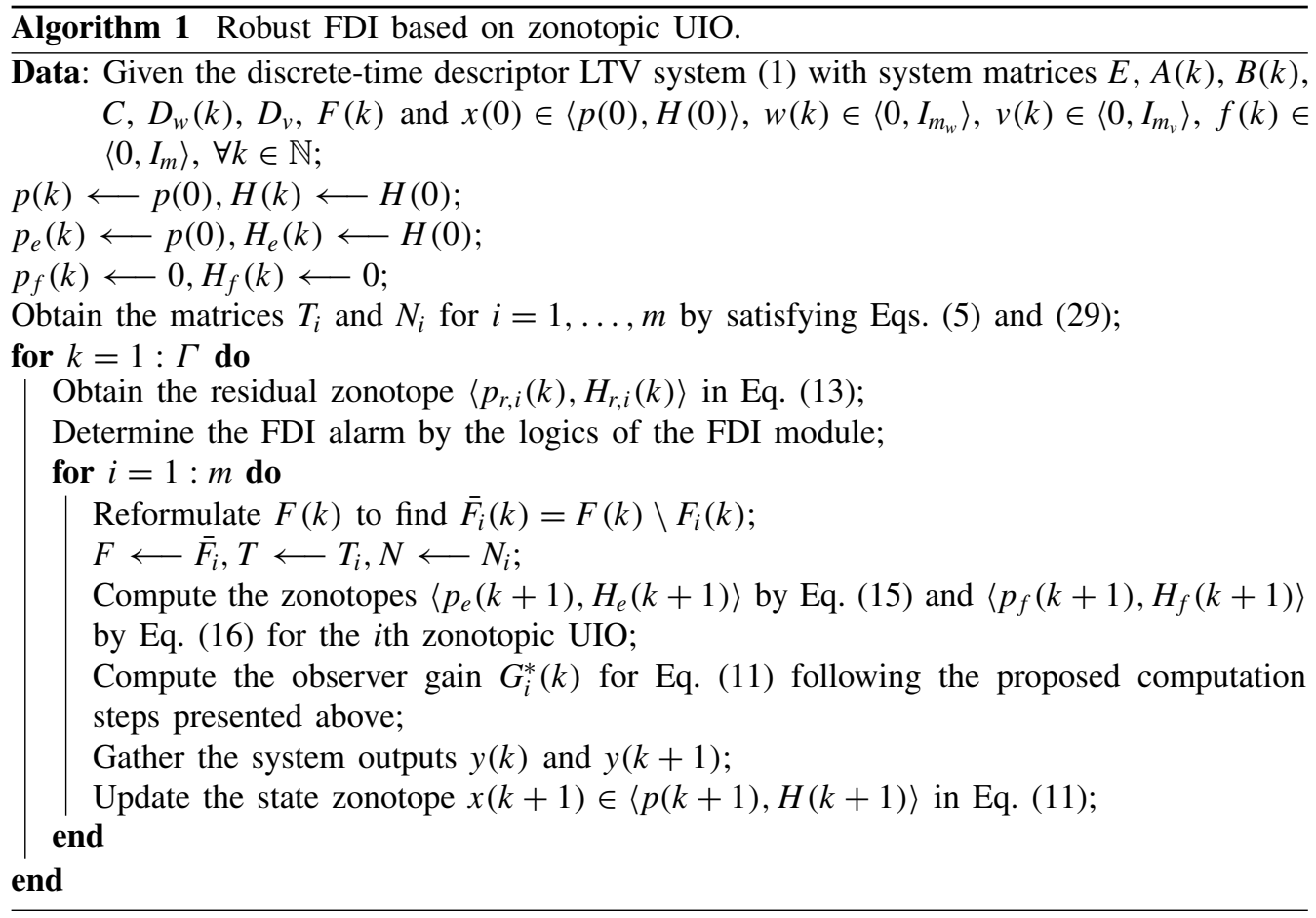

and the initial state $x(0)=[2,2,3,3.125]^{\top}$ is assumed to be bounded by the zonotope $x(0) \in\langle p(0), H(0)\rangle$, where $p(0)=x(0)$ and $H(0)=0.1 I_{4}$. The weighting matrices $W_{1}$ and $W_{2}$ for designing the FD observer gain are chosen to be identity matrices of appropriate dimensions. The input signal $u$ is set as $u(k)=2, \forall k \in \mathbb{N}$. For the reduction operator $\downarrow_{q, W}(\cdot), q$ and $W$ are set respectively as $q=20$ and $W=I$. With constant matrices $E$ and $C$, by satisfying the condition (5), we consider one solution of constant matrices $T$ and $N$ as follows:

$T=\left[\begin{array}{cccc}1 & 0 & 0 & 0 \\ 0 & 0.5 & 0 & 0 \\ 0 & 0 & 0.5 & 0 \\ 0 & 0 & 0 & 1\end{array}\right], N=\left[\begin{array}{ccc}0 & 0 & 0 \\ 0.5 & 0 & 0 \\ 0 & 0.5 & 0 \\ 0 & 0 & 1\end{array}\right]$.

The simulation has been carried out in MATLAB for 100 sampling time steps. With this example, we compute the time-varying Kalman gain $\bar{G}(k)$ (following Theorem 3 ) and the designed FD observer gain $G^{*}(k)$ (following Theorem 5) at each time step. Since the system has three measurement outputs, the residual zonotope $\left\langle p_{r}(k), H_{r}(k)\right\rangle, \forall k \in \mathbb{N}$ is in a 3-dimensional space. Therefore, the interval hull (see Definition 2) of the residual zonotope is used to plot the individual residual bounds $r_{i}(k) \in\left[\underline{r_{i}}(k), \overline{r_{i}}(k)\right]$ for $r_{i}(k) \in \mathbb{R}^{n_{y}}$, where

$\underline{r_{i}}(k)=p_{r, i}(k)-r s\left(H_{r}(k)\right)_{i, i}, i=1, \ldots, n_{y}, \forall k \in \mathbb{N}$,

$\overline{r_{i}}(k)=p_{r, i}(k)+r s\left(H_{r}(k)\right)_{i, i}, i=1, \ldots, n_{y}, \forall k \in \mathbb{N}$.

Consider a step actuator fault $f(k)=0.3, k>=30$. The comparative results of the residuals and their lower and upper bounds are shown in Fig. 2. From these plots, it is shown that 


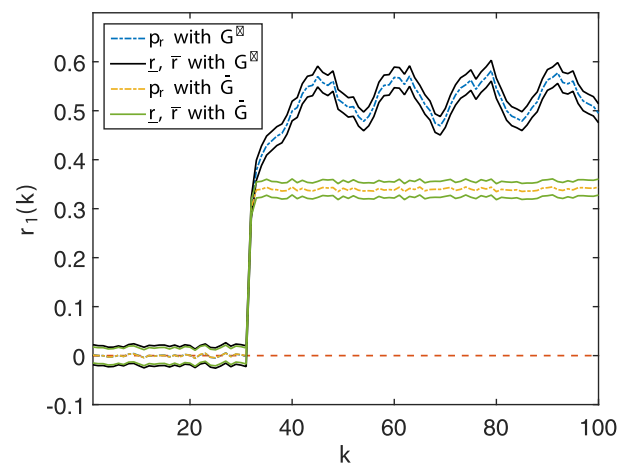

(a) $r_{1}(k)$

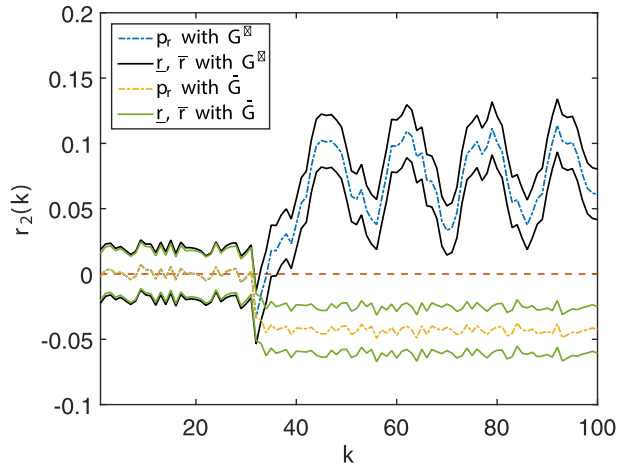

(b) $r_{2}(k)$

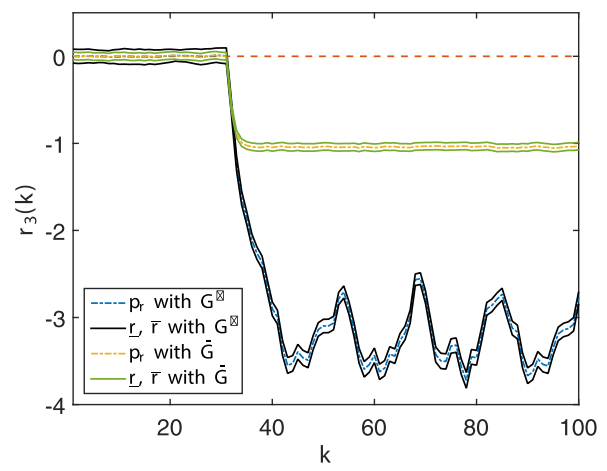

(c) $r_{3}(k)$

Fig. 2. The comparison of generated residual results.

Table 1

Minimum detectable fault with the Kalman and FD gains.

\begin{tabular}{llll}
\hline & $\bar{G}(k)$ & $G^{*}(k)$ & Improvement \\
\hline Minimal detectable fault & 0.0135 & 0.0089 & $51.69 \%$ \\
\hline
\end{tabular}

when no fault occurred $(f(k)=0, k<30)$, zero is inside all the residual bounds, that is also inside the residual zonotope. Besides, the bounds with the Kalman gain $\bar{G}(k)$ are tighter than those obtained with the FD observer gain $G^{*}(k)$. This is because the objective of the Kalman gain design is to minimize the effects of uncertainties. On the other hand, when the system is affected by actuator fault $f(k)=0.3, k>=30$, the residual bounds obtained with $G^{*}(k)$ do not contain zero that according to Eq. (14) the fault is detected thus being more sensitive with respect to the occurred fault. This improved fault sensitivity will be useful when faults with small magnitude occur.

Table 1 presents the minimal detectable faults of this example with two observer gains obtained in simulation. It is shown that the observer with $G^{*}$ is able to detect smaller faults when the fault sensitivity is considered. The trade-off between robustness to uncertainties and sensitivity to faults is improved by using $G^{*}(k)$. 
Unknown input decoupling for robust FDI strategy.

\begin{tabular}{llcc}
\hline & $T, N$ & $f_{1}$ & $f_{2}$ \\
\hline Zonotopic UIO 1 & $T_{1}, N_{1}$ & $\times$ & $\times$ \\
Zonotopic UIO 2 & $T_{2}, N_{2}$ & $\times$ & \\
\hline
\end{tabular}

\subsection{The chemical mixing system}

We consider a chemical mixing system in [39] to test the proposed robust FDI strategy based on a bank of zonotopic UIOs. In this system, there are two chemical mixture tanks, two valves and several pipes. Chemical concentrations and outflows of two output pipes are chosen as states and disturbances for each state are considered. The flow rates from two valves are chosen as inputs and actuator faults refer to these two valves. We use the Euler method with sampling time $t_{s}=0.1 \mathrm{~s}$ to discretize the descriptor model of the chemical mixing system described by Eq. (1) with

$E=\left[\begin{array}{llll}1 & 0 & 0 & 0 \\ 0 & 0 & 0 & 0 \\ 0 & 0 & 1 & 0 \\ 0 & 0 & 0 & 0\end{array}\right], A=\left[\begin{array}{cccc}0.9625 & 0.0067 & 0 & 0 \\ 0 & -0.1 & 0 & 0 \\ 0.03 & 0.0533 & 0.95 & -0.004 \\ 0 & 0.1 & 0 & -0.1\end{array}\right], B=\left[\begin{array}{cc}0.01 & 0 \\ 0.1 & 0 \\ 0 & 0.002 \\ 0 & 0.1\end{array}\right]$,

$C=\left[\begin{array}{llll}0 & 1 & 0 & 0 \\ 0 & 0 & 1 & 0 \\ 0 & 0 & 0 & 1\end{array}\right], D_{w}=0.001 I_{4}, D_{v}=0.01 I_{3}, F=B$

and the initial state $x(0)=[0.5,0,0.5,0]^{\top}$ is assumed to be bounded by the zonotope $x(0) \in\langle p(0), H(0)\rangle$, where $p(0)=x(0)$ and $H(0)=0.001 I_{4}$. The weighting matrices $W_{1}$ and $W_{2}$ for designing the FD observer gain are also chosen to be identity matrices of appropriate dimensions. The input signal $u$ is set as $u(k)=[4 \sin (0.3 k)+5,5]^{\top}, \forall k \in \mathbb{N}$. For the reduction operator $\downarrow_{q, W}(\cdot), q$ and $W$ are set respectively as $q=20$ and $W=I$. Taking into account that this system has two actuators, two zonotopic UIOs are used. For implementing robust FDI strategy, the actuator faults are considered to be unknown inputs for each zonotopic UIO and the unknown input decoupling strategy is described in Table 2.

By satisfying the conditions in Eqs. (5) and (29), and considering the strategy in Table 2, we have

$\begin{aligned} T_{1} & =\left[\begin{array}{llcl}1 & 1 & 0 & 1 \\ 0 & 1 & 0 & 1 \\ 0 & 1 & 0.5 & 1 \\ 0 & 1 & 0 & 1\end{array}\right], N_{1}=\left[\begin{array}{ccc}0 & 0 & 0 \\ 1 & 0 & 0 \\ 0 & 0.5 & 0 \\ 0 & 0 & 1\end{array}\right], \\ T_{2} & =\left[\begin{array}{llll}1 & 23.4588 & 0.1777 & 0.0032 \\ 0 & 23.4588 & 0.1777 & 0.0032 \\ 0 & 23.4588 & 0.1777 & 0.0032 \\ 0 & 23.4588 & 0.1777 & 0.0032\end{array}\right], N_{2}=\left[\begin{array}{ccc}0 & -0.1777 & 0 \\ 1 & -0.1777 & 0 \\ 0 & 0.8223 & 0 \\ 0 & -0.1777 & 1\end{array}\right] .\end{aligned}$

The zonotopic UIO 1 is designed with $T_{1}$ and $N_{1}$. Besides, the sensitivity to the second actuator fault is considered such that $\bar{F}_{1}(k)=[0,0,0.02,1]^{\top}$. The zonotopic UIO 2 is designed with $T_{2}$ and $N_{2}$. Because the effect of the second actuator fault is removed by using 

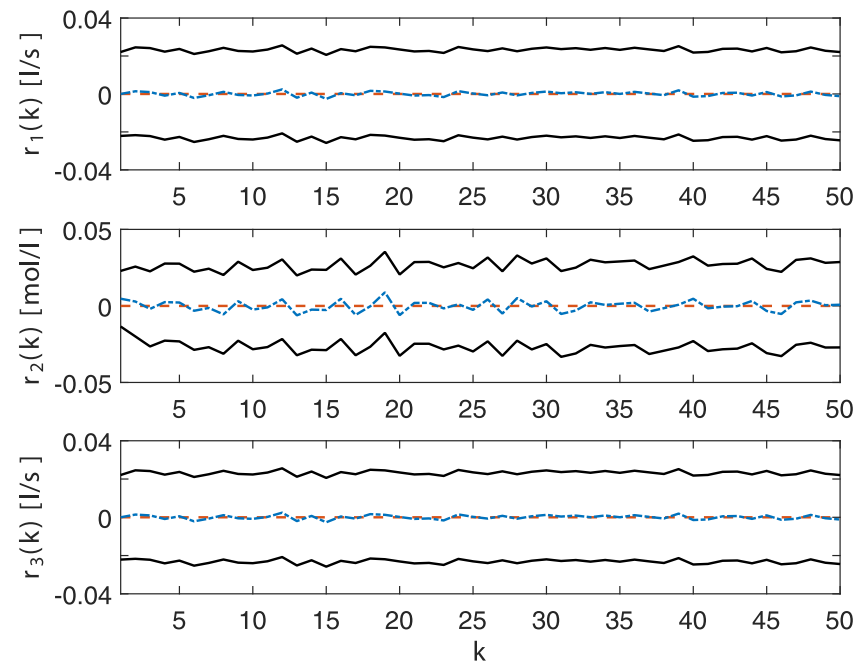

(a) Zonotopic UIO 1
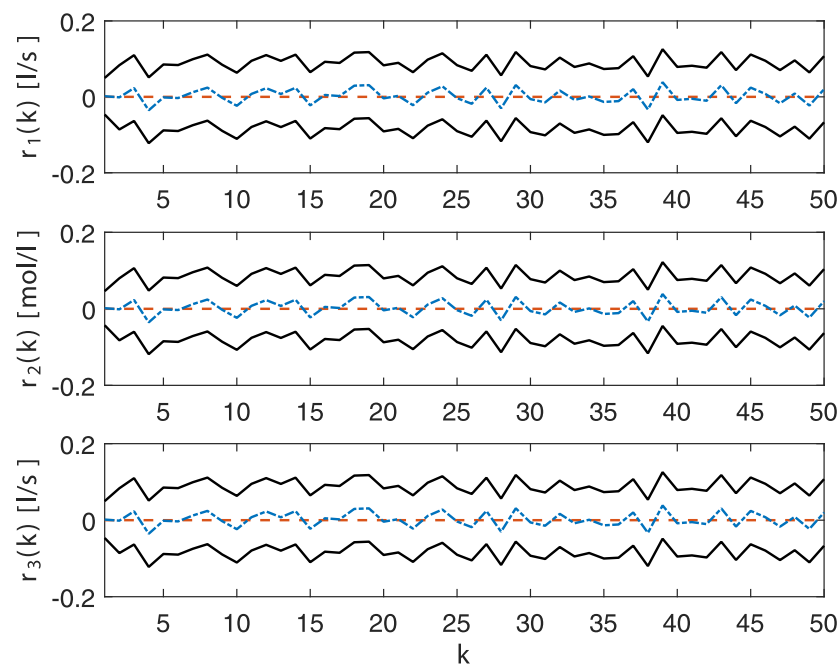

(b) Zonotopic UIO 2

Fig. 3. The FDI result of the chemical mixing system without occurred faults.

the unknown input decoupling, this observer is designed to be sensitive to the first actuator fault.

Simulations with different abrupt step actuator faults have been carried out. In Fig. 3, it is shown that the coordinate origin is inside all the residual bounds of zonotopic UIOs 1 and 2, that is inside the residual zonotopes corresponding to zonotopic UIOs 1 and 2, which implies that no fault has occurred. In Fig. 4, it can be seen that for both zonotopic UIOs, the coordinate origin is not inside all the residual bounds after 20 sampling time steps. Based 

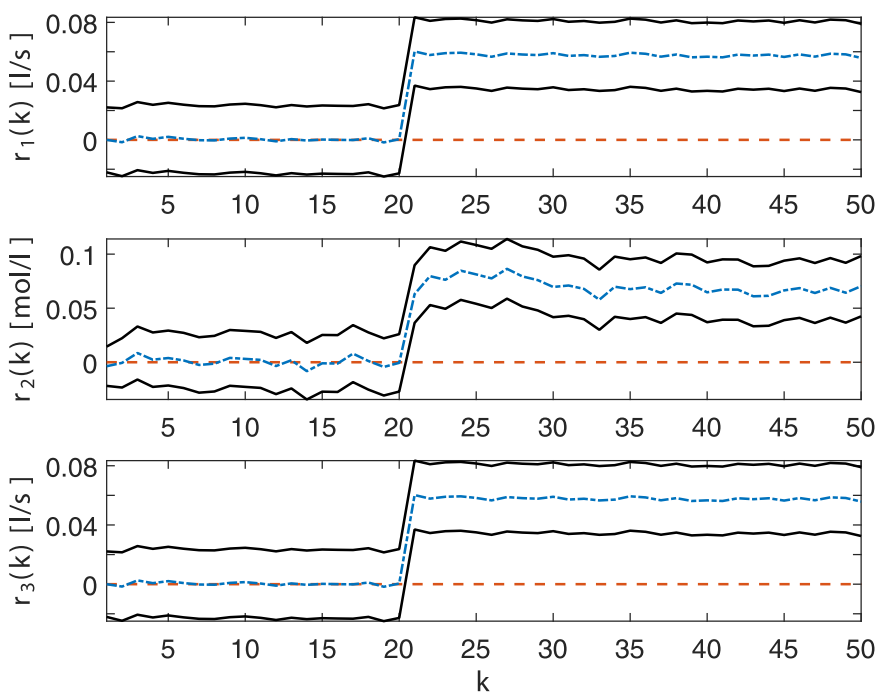

(a) Zonotopic UIO 1
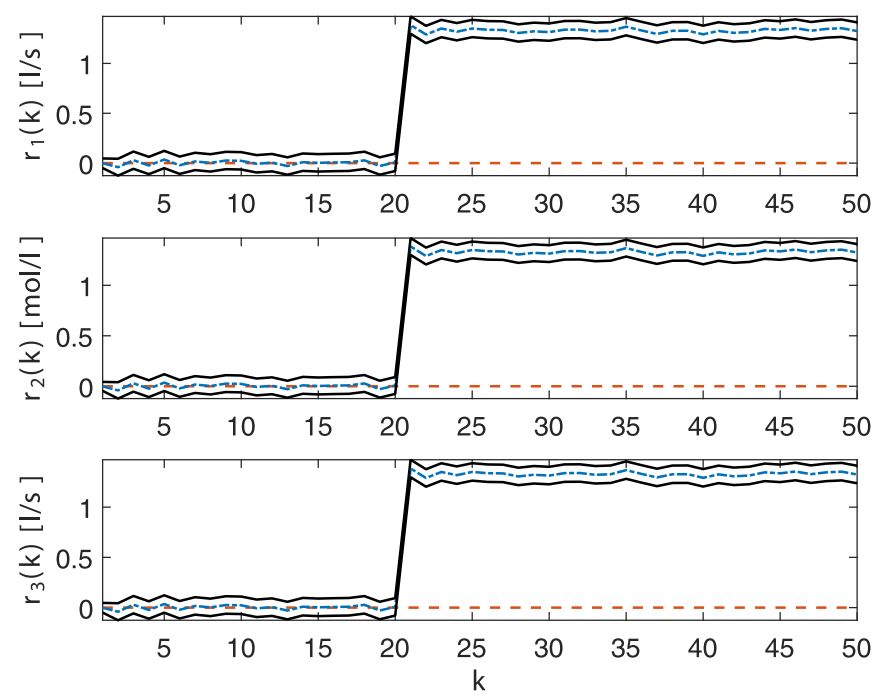

(b) Zonotopic UIO 2

Fig. 4. The FDI result of the chemical mixing system with first actuator fault.

on Table 2 and the designed FDI strategy, the first actuator fault is detected at time step 21. Fig. 5 shows that the coordinate origin is always inside the residual bounds of zonotopic UIO 2. Besides, after 20 sampling time steps the coordinate origin is not inside the residual bounds of zonotopic UIO 1. According to the proposed FDI strategy, the second actuator fault is detected at time step 21 . 

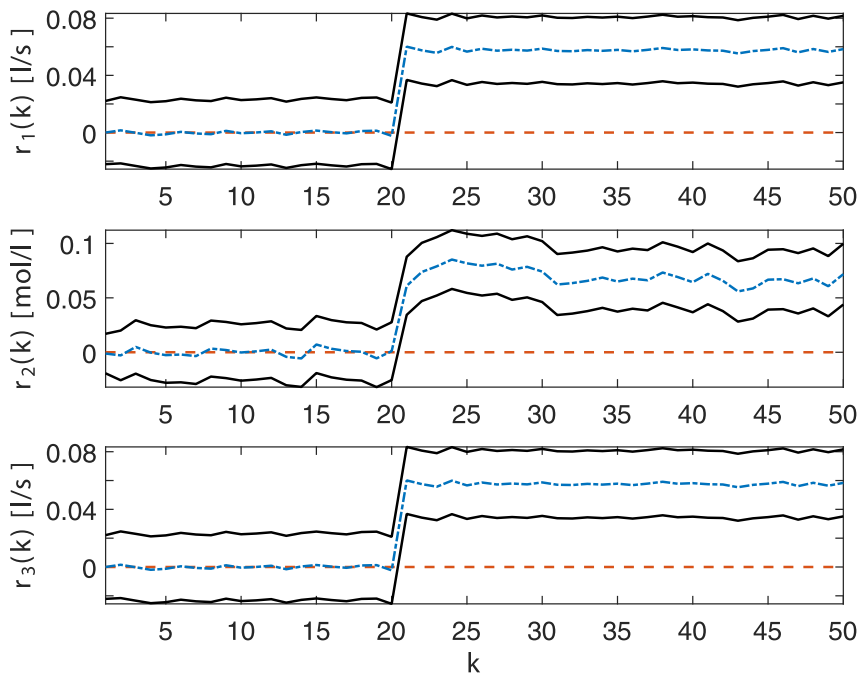

(a) Zonotopic UIO 1
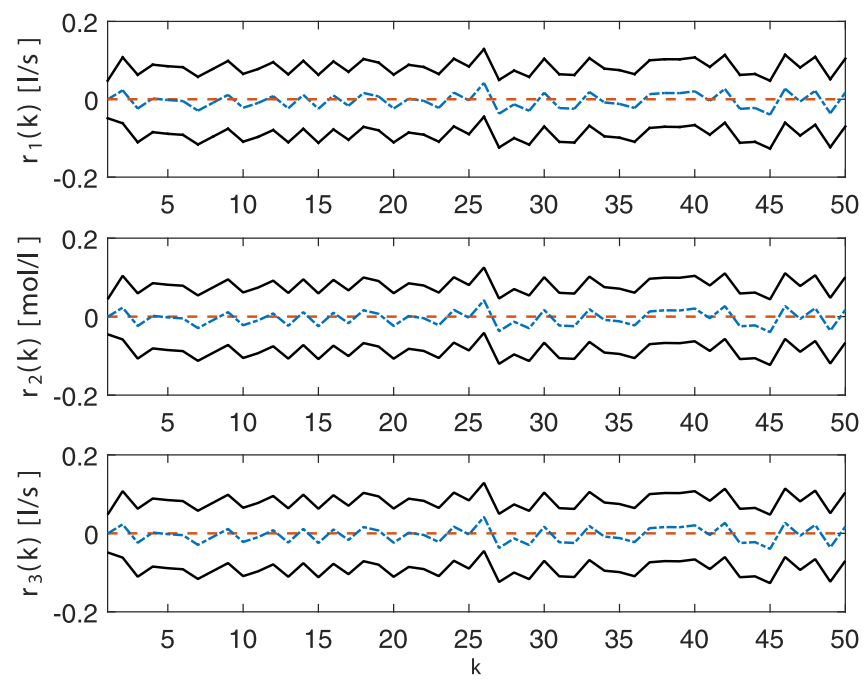

(b) Zonotopic UIO 2

Fig. 5. The FDI result of the chemical mixing system with second actuator fault.

\section{Conclusion}

In this paper, we have proposed a robust FDI based on zonotopic UIOs for discretetime descriptor LTV systems. The time-varying observer gains have been designed based on different objectives. When only the effect of uncertainties are minimized, the optimal Kalman gain is given, while, in order to design an FD observer gain, a performance criterion is defined to consider robustness against uncertainties and sensitivity to faults. An algebraic solution of 
the FD observer gain based on this performance criterion is developed. The proposed scheme is extended to FI by building a bank of zonotopic UIOs. For each zonotopic UIO, a single actuator fault is considered as an unknown input. By means of the transformation matrices, the unknown input can be decoupled. Hence, this zonotopic UIO is designed to be sensitive to the remaining faults that are not decoupled. Therefore, the FDI strategy is implemented by testing whether the coordinate origin is in the residual zonotopes generated by a bank of zonotopic UIOs. Through two case studies, we have shown the effectiveness of the FD observer gain design procedure and the robust FDI strategy. The considered descriptor systems are common in network dynamics, such as power grids, energy or water distribution networks, etc. Hence, the proposed FDI strategy is useful for guaranteeing the safety and reliability of these networks and could be integrated into a fault-tolerant control scheme.

\section{Acknowledgments}

This work was partially funded by the Spanish State Research Agency (AEI) and the European Regional Development Fund (ERFD) through the project DEOCS (ref. DPI201676493-C3-3-R) and the FPI grant (ref. BES-2014-068319), and by the Basic Research Project of Shenzhen under the grant (ref. JCYJ20170412171459177).

\section{References}

[1] M. Blanke, M. Kinnaert, J. Lunze, M. Staroswiecki, Diagnosis and Fault-Tolerant Control, Springer, Berlin Heidelberg, Germany, 2016.

[2] S. Ding, Model-Based Fault Diagnosis Techniques, Springer, London, UK, 2013.

[3] A. Varga, Solving Fault Diagnosis Problems, Springer, 2017.

[4] J. Liu, W. Luo, X. Yang, L. Wu, Robust model-based fault diagnosis for PEM fuel cell air-feed system, IEEE Trans. Ind. Electron. 63 (5) (2016) 3261-3270.

[5] H. Li, Y. Gao, P. Shi, H. Lam, Observer-based fault detection for nonlinear systems with sensor fault and limited communication capacity, IEEE Trans. Autom. Control 61 (9) (2016) 2745-2751.

[6] H. Li, Y. Gao, L. Wu, H. Lam, Fault detection for T-S fuzzy time-delay systems: delta operator and input-output methods, IEEE Trans. Cybern. 45 (2) (2015) 229-241.

[7] J. Chen, R. Patton, Robust Model-Based Fault Diagnosis for Dynamic Systems, Springer, New York, USA, 2012.

[8] M. Chadli, A. Abdo, S. Ding, $H_{-} / H_{\infty}$ fault detection filter design for discrete-time Takagi-Sugeno fuzzy system, Automatica 49 (7) (2013) 1996-2005.

[9] D. Ichalal, B. Marx, J. Ragot, D. Maquin, Fault detection, isolation and estimation for Takagi-Sugeno nonlinear systems, J. Frankl. Inst. 351 (7) (2014) 3651-3676.

[10] T. Lee, K.S. Park, Robust fault detection observer design under fault sensitivity constraints, J. Frankl. Inst. 352 (5) (2015) 1791-1810.

[11] S. Raka, C. Combastel, Fault detection based on robust adaptive thresholds: a dynamic interval approach, Annu. Rev. Control 37 (1) (2013) 119-128.

[12] F. Xu, V. Puig, C. Ocampo-Martinez, F. Stoican, S. Olaru, Actuator-fault detection and isolation based on set-theoretic approaches, J. Process Control 24 (6) (2014) 947-956.

[13] F. Blanchini, D. Casagrande, G. Giordano, S. Miani, S. Olaru, V. Reppa, Active fault isolation: a duality-based approach via convex programming, SIAM J. Control Optim. 55 (3) (2017) 1619-1640.

[14] V. Puig, J. Quevedo, T. Escobet, F. Nejjari, S. de las Heras, Passive robust fault detection of dynamic processes using interval models, IEEE Trans. Control Syst. Technol. 16 (5) (2008) 1083-1089.

[15] Y. Wang, V. Puig, Zonotopic extended Kalman filter and fault detection of discrete-time nonlinear systems applied to a quadrotor helicopter, in: Proceedings of the Third Conference on Control and Fault-Tolerant Systems (SysTol), 2016, pp. 367-372. 
[16] J. Scott, D. Raimondo, G. Roberto Marseglia, R. Braatz, Constrained zonotopes: a new tool for set-based estimation and fault detection, Automatica 69 (2016) 126-136.

[17] Y. Wang, V. Puig, G. Cembrano, Set-membership approach and Kalman observer based on zonotopes for discrete-time descriptor systems, Automatica 93 (2018) 435-443.

[18] Y. Wang, M. Zhou, V. Puig, G. Cembrano, Z. Wang, Zonotopic fault detection observer with $H_{-}$performance, in: Proceedings of the 2017 Chinese Control Conference (CCC), 2017, pp. 7230-7235.

[19] M. Hou, P.C. Muller, Design of observers for linear systems with unknown inputs, IEEE Trans. Autom. Control 37 (6) (1992) 871-875.

[20] M. Chadli, H. Karimi, Robust observer design for unknown inputs Takagi-Sugeno models, IEEE Trans. Fuzzy Syst. 21 (1) (2013) 158-164.

[21] H. Hassani, J. Zarei, M. Chadli, J. Qiu, Unknown input observer design for interval Type-2 T-S fuzzy systems with immeasurable premise variables, IEEE Trans. Cybern. 47 (9) (2017) 2639-2650.

[22] D. Rotondo, M. Witczak, V. Puig, F. Nejjari, M. Pazera, Robust unknown input observer for state and fault estimation in discrete-time Takagi-Sugeno systems, Int. J. Syst. Sci. 47 (14) (2016) 3409-3424.

[23] D. Koenig, Unknown input proportional multiple-integral observer design for linear descriptor systems: application to state and fault estimation, IEEE Trans. Autom. Control 50 (2) (2005) 212-217.

[24] F. Xu, J. Tan, X. Wang, V. Puig, B. Liang, B. Yuan, A novel design of unknown input observers using set-theoretic methods for robust fault detection, in: Proceedings of the 2016 American Control Conference (ACC), 2016, pp. 5957-5961.

[25] F. Xu, J. Tan, X. Wang, V. Puig, B. Liang, B. Yuan, H. Liu, Generalized set-theoretic unknown input observer for LPV systems with application to state estimation and robust fault detection, Int. J. Robust Nonlinear Control 27 (17) (2017) 3812-3832.

[26] F. Xu, J. Tan, X. Wang, V. Puig, B. Liang, B. Yuan, Mixed active/passive robust fault detection and isolation using set-theoretic unknown input observers, IEEE Trans. Autom. Sci. Eng. 15 (2) (2018) 863-871.

[27] Y. Wang, V. Puig, G. Cembrano, Non-linear economic model predictive control of water distribution networks, J. Process Control 56 (2017) 23-34.

[28] L. Biegler, S. Campbell, V. Mehrmann, Control and Optimization with Differential-Algebraic Constraints, Society for Industrial and Applied Mathematics, 2012.

[29] B. Stevens, F. Lewis, E. Johnson, Aircraft Control and Simulation: Dynamics, Controls Design, and Autonomous Systems, Wiley-Blackwell, 2016.

[30] R. Riaza, Differential-Algebraic Systems: Analytical Aspects and Circuit Applications, World Scientific Publishing Company, 2008.

[31] C. Combastel, A state bounding observer for uncertain non-linear continuous-time systems based on zonotopes, in: Proceedings of the 2005 IEEE Conference on Decision and Control and European Control Conference (CDC-ECC), 2005, pp. 7228-7234.

[32] L. Dai, Singular Control Systems, Springer, Berlin Heidelberg, Germany, 1989.

[33] B. Boulkroune, S. Halabi, A. Zemouche, $H_{-} / H_{\infty}$ fault detection filter for a class of nonlinear descriptor systems, Int. J. Control 86 (2) (2013) 253-262.

[34] Z. Wang, P. Shi, C. Lim, $H_{-} / H_{\infty}$ fault detection observer in finite frequency domain for linear parameter-varying descriptor systems, Automatica 86 (2017) 38-45.

[35] Z. Wang, M. Rodrigues, D. Theilliol, Y. Shen, Fault estimation filter design for discrete-time descriptor systems, IET Control Theory Appl. 9 (10) (2015) 1587-1594.

[36] S. Chang, W. You, P. Hsu, Design of general structured observers for linear systems with unknown inputs, J. Frankl. Inst. 334 (2) (1997) 213-232.

[37] M. Gupta, N. Tomar, S. Bhaumik, Full- and reduced-order observer design for rectangular descriptor systems with unknown inputs, J. Frankl. Inst. 352 (3) (2015) 1250-1264.

[38] C. Combastel, Zonotopes and Kalman observers: gain optimality under distinct uncertainty paradigms and robust convergence, Automatica 55 (2015) 265-273.

[39] T. Yeu, H. Kim, S. Kawaji, Fault detection, isolation and reconstruction for descriptor systems, Asian J. Control 7 (4) (2005) 356-367. 